IJBPAS, October, Special Issue, 2021, 10(10): 523-545

ISSN: $2277-4998$

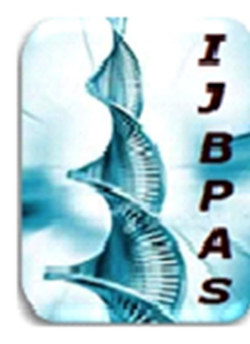

International Journal of Biology, Pharmacy and Allied Sciences (IJBPAS) 'A Bridge Botween caboratory and Qender'

Www.ijbpas.com

\title{
DESIGN, DEVELOPMENT AND EVALUATION OF ORAL SUPER- SMEDDS FORMULATIONS OF UDENAFIL
}

\section{MEGHANA BABAR* AND JYOTI GULABANI}

1: Departments of Pharmaceutics, Dr L. H. Hiranandani College of Pharmacy, Ulhasnagar- 421003

*Corresponding Author: E Mail: meghana.babar@dlhhcop.org

Received 27 $7^{\text {th }}$ April 2021; Revised 25 ${ }^{\text {th }}$ June 2021; Accepted $1^{\text {st }}$ Aug. 2021; Available online $1^{\text {st }}$ Oct. 2021

https://doi.org/10.31032/IJBPAS/2021/10.10.1047

\begin{abstract}
Udenafil is a BCS class II drug with very low aqueous solubility (approximately 0.0798 $\mathrm{mg} / \mathrm{ml}$ ) and is used in urology to treat erectile dysfunction. It shows low oral bioavailability (approximately 38\%) due to lower solubility and intestinal first pass metabolism. A drug having low solubility in gastro-intestinal tract cannot be absorbed completely and needs to be formulated in a special formulation.Current work was carried out to develop and evaluate supersaturated self emulsifying drug delivery system (Super-SMEDDS) of udenafil in order to attain enhanced solubility and dissolution properties with improved oral bioavailability. The solubility of Udenafil was checked in different oils, surfactants, and cosurfactants and ternary phase diagrams were constructed to evaluate the microemulsion domain. The Udenafil SMEDDS was prepared using Oleic acid (oil), Cremophor RH40 (surfactant), and polyethylene glycol 400 (cosurfactant). In order to produce the formulation comparable with the available marketed tablet the Super-SMEDDS formulations were developed where drug loading was increased to $100 \mathrm{mg}$ in preconcentrates. Developed formulations were evaluated for particle size distribution, zeta potential, polydispersity index, and subjected to in vitro release studies and ex vivo permeability studies. The optimized formulation produced fine microemulsion with globule size of $158.8 \pm 2.15 \mathrm{~nm}$, polydispersibility index in range of $0.369 \pm 0.06$ and zeta potential value of $-37 \pm 4 \mathrm{mV}$. Freeze thaw cycling, centrifugation test, dilution study showed that optimized formulation is thermodynamically stable. In vitro dissolution studies of Super-SMEDDS against UDL API and Udzire tablet $(100 \mathrm{mg}$ ) carried out in dissolution media $0.1 \mathrm{~N} \mathrm{HCl}$ and $\mathrm{pH} 6.8$ showed a
\end{abstract}


dramatic improvement in the in vitro dissolution profiles. Results of ex vivo studies indicate nearly three folds improvement in permeability of drug solution. Developed formulations were found to be promising and may result in improved therapeutic performance.

Keywords: Super-SMEDDS, Udenafil, Erectile dysfunction, Cremophor RH40, Oleic acid, polyethylene glycol 400

Abbreviations: Supersaturated self emulsifying drug delivery system (Super-SMEDDS), Udenafil(UDL), Simulated gastric fluid (SGF), Simulated intestinal fluid (SIF), \% transmittance (\%T), Oleic Acid (OLA), Krebs-Ringer-phosphate-buffer (KRPB), Liquid SMEDDS (LSMEDDS), Cremophor RH40 Cr-RH40, Polyethylene Glycol (PEG 400)

\section{INTRODUCTION}

It has been researched that between $40 \%$ and $70 \%$ of all new chemical entities identified in drug discovery programs are insufficiently soluble in aqueous media to allow adequate and reproducible absorption from gastrointestinal tract, following oral administration [1]. Lipid-based drug delivery systems incorporating such NCEs have gained much importance in the recent years due to their ability to improve the solubility and absorption in vivo [2]. These systems increase absorption from the gastrointestinal tract by accelerating the dissolution process, facilitating the formation of solubilized phases by reduction of particle size to the molecular level, changing drug uptake, efflux and disposition by altering enterocyte-based transport and enhancing drug transport to the systemic circulation via intestinal lymphatic system [3]. Amongst these systems SMEDDS are principally useful for oral delivery of lipophilic and poorly water-soluble drugs. They form transparent microemulsion with emulsion droplets ranging between 100 to $250 \mathrm{~nm} \mathrm{[4].} \mathrm{Inspite}$ and despite of of possessing many advantages like improvement in oral bioavailability, ease of manufacture and scale-up, reduction in inter-subject and intra-subject variability and food effects, etc., limited solubility of many drugs in SMEDDS is been proved as the major obstacle for the broader utilisation of SMEDDS often requiring inconvenient dosing of multiple units [5]. Traditionally, the dose of a drug that can be administered in SMEDDS is limited by the solubility of the drug in the preconcentrate. Thus, in order to avoid the administration of multiple units of a dosage form (usually soft gelatin or liquid filled hard gelatin capsules), a drug should exhibit adequate solubility in the SMEDDS preconcentrates to allow sufficient and convenient dosing for a desired therapeutic intervention. However, the solubility of drugs in the preconcentrates is oftenrestricted, which 
may limit the usefulness of lipid-based delivery systems. Novel supersaturated SMEDDS super-SMEDDS) allowed the dosing of single units as the drug load in the super- SMEDDS substantially increased compared with conventional SMEDDS [6].

The super-SMEDDS are physically stable and bio- pharmaceutically comparable and, in some cases, superior to conventional SMEDDS. Main advantage Of SuperSMEDDS is that it improves patient compliance by reducing multiple-daily dosing regimen to once daily dosing regimen.

Udenafil(UDL) is a BCS class II drug with very low aqueous solubility (approximately $0.0798 \mathrm{mg} / \mathrm{ml}$ ). It shows low oral bioavailability (approximately 38\%) due to decreased solubility and intestinal first pass metabolism. A drug having low solubility in gastro-intestinal tract cannot be absorbed completely and needs to be formulated in a special formulation [7].

Hence in current study Super-SMEDDS formulations were investigated as this delivery system overcomes the rate limiting properties of udenafil i.e low aqueous solubility, intestinal first pass effect thus increasing its oral bioavailability.

\section{MATERIALS \& METHODS}

\section{Materials}

Udenafil was a generous gift from Watson Pharma Pvt. Ltd., Maharashtra,
India.Isopropyl myristate, Captex 355, Captex 200, Captex 355 EP/NF, Labrafac PG, Capmul MCM NFCapmul MCM C8, Capmul MCM EP was obtained as gift sample from BASF, Mumbai India. Oleic acid, Corn oil, Sunflower oil, Olive oil, Castor oil, Coconut oil, Soyabean oil, Arachis oil and Tea tree oilwere purchases from S. D. Fine Chemicals.

\section{Methods}

Ultavioiet Spectroscopy Method for Estimation of $U D L$

Standard stock solution $(1000 \mu \mathrm{g} / \mathrm{ml})$ was suitably diluted with Methanol to obtain concentrations ranging from $5-35 \mu \mathrm{g} / \mathrm{ml}$. Absorbance of these solutions was measured at $291.8 \mathrm{~nm}$, calibration curve was obtained by plotting graph between concentration and absorbance, and regression coefficient was estimated. UV spectrophotometric analysis was also carried out in buffer solutions of $\mathrm{pH} 6.8,4$, 1.2 and water.

Solubility Study of UDL in Various Oils, Water and Buffer Solutions

UDL Solubility was determined by modifying the routine shake flask method which has been explained by various researchers. The developed method was divided in two stages. In first stage approximate solubility of UDL was determined by visual inspection. In a vial containing $1 \mathrm{gm}$ of prewarmed oils, 
accurately weighed known quantities of UDL were added till the complete saturation of vehicle. The amount of UDL causing satutation of each oil was taken as its approximate solubility in the respective oil. In second stage, only few promising oils showing appreciable solubility were further studied for quantitative estimation of UDL. The quantitative estimation of solubility of UDL in water, $0.1 \mathrm{~N} \mathrm{HCL}$, Phosphate buffer and Acetate buffer was also performed.

\section{Emulsification Efficiency StudySelection Of Surfactant and Cosurfactant}

Different surfactants were screened for its emulsification ability for selected oil phase i.e., Oleic acid. Initially mixtures were prepared in 1:1 ratio and Emulsification efficiency of surfactants was screened based on $\%$ transmittance and emulsification ability. The mixture was then gently heated at $40-50{ }^{\circ} \mathrm{C}$ for homogenizing the components. Since observed \% transmittance was less than $70 \%$, additional trials with 1:3 ratio were investigated. The selected oil i.e.,Oleic acid (OLA) and selected surfactant Cremophor RH-40 were further used for screening different co-surfactant. For this selection the surfactant mixture was prepared by mixing surfactant and co-surfactant in 3:1 ratio and was assessed for the relative efficiency of the co-surfactant to improve the emulsification ability of selected surfactant.

\section{Construction of Ternary Phase Diagrams}

Ternary phase diagram of oil, surfactant, co-surfactant was plotted by using flask inversion method. The concentrations of each of component was decided on the basis of requirements, stated by pouton, for the spontaneously emulsifying systems i.e., 25 to $70 \%$ oily phase, 30 to $75 \%$ surfactant and 0 to $30 \%$ co-surfactant. Numerous systems were prepared by changing the ratio of each component. Based on the solubility study and emulsification efficiency, self-emulsifying system OLA: Cr-RH 40: PEG 400 was selected to produce ternary phase diagram. Forty-five different systems of this combination were prepared by varying concentration of Oil, surfactant and co-surfactant. All the systems were evaluated for formation of microemulsion by diluting $50 \mathrm{mg}$ of each mixture by $50 \mathrm{ml}$ of Distilled water in volumetric flask, flask inversions were performed to facilitate microemulsion formation. The obtained emulsion was allowed to stand for $2 \mathrm{hr}$ and their \% transmittance was noted at $638.2 \mathrm{~nm}$ by using UV-Visible spectrophotometer against distilled water as blank. Systems that required less than 10 flask inversions (FI) to produce clear and transparent or slightly bluish solutions with more than 90 
$\%$ transmittance were considered as microemulsion producing systems, while the systems which produced white or nonclear turbid emulsion (less than 90\% T) are considered as coarse emulsion producing systems, and the systems which did not disperse globules and remained turbid $(>70 \% \mathrm{~T})$ even after more than 20 flask inversions are considered as systems incapable to produce emulsion, hence noted as no emulsion producing systems. All systems were used to plot ternary phase diagram and microemulsion region of system was identified from obtained phase diagram. Ternary phase diagram was constructed with the help of Triplot software.

Effect of UDL loading and $p H$ of the aqueous phase on selected system OLA: Cr-RH 40: PEG 400

The drug as well as $\mathrm{pH}$ of the vehicle have considerable influence on the phase behavior of the spontaneously emulsifying systems. In the view of this effect, the effect of UDL and $\mathrm{pH}$ of the aqueous phase on the phase behavior and the area of microemulsion formation was studied. To investigate the effect, UDL loaded OLA was prepared by dissolving UDL in OLA and used as oily phase while constructing phase diagram. The two different phase diagrams were constructed in the similar fashion explained above by replacing distilled water by $0.1 \mathrm{~N}$ HCL and phosphate buffer $\mathrm{pH} 6.8$ as a vehicle. Ternary phase diagram of oil, surfactant, co-surfactant were plotted by using flask inversion method. All the systems were evaluated for formation of microemulsion by diluting 50 $\mathrm{mg}$ of each mixture by $50 \mathrm{ml}$ of buffer in volumetric flask, flask inversions were performed to facilitate microemulsion formation. All systems were used for plotting ternary phase diagram and region of system capable to produce microemulsion was identified from obtained phase diagram.

Formulation and development of LSMEDDS

\section{Preparation of Liquid SMEDDS (L-} SMEDDS)

UDL was added with a fixed dose of $50 \mathrm{mg}$ formulation of LSMEDDS was carried out in $10 \mathrm{ml}$ glass vial in which specified amount of oleic acid and UDL was added on weight basis, to facilitate the complete solubility of drug, this mixture was warmed at $40-50{ }^{\circ} \mathrm{C}$ in water bath. Respective quantity of Cr-RH 40 and PEG 400 was added to this mixture, warmed on water bath and homogenized by cyclo-mixer for 5-10 mins resulting in clear isotropic system. These formulations of L-SMEDDS containing $50 \mathrm{mg}$ of UDL were stored at room temperature for the further study.

Optimization of L-SMEDDS of UDL 


\section{- Freeze thaw cycling}

To access stability of microemulsion selected betched were placed in at $40^{\circ} \mathrm{C}$ for 24 hours in incubator and followed by 24 hours at $5^{\circ} \mathrm{C}$ in refrigerator, the cycle was repeated for 3 times and after each cycle the formulations were visually observed for any sign of phase separation and/or precipitation of drug. Formulation batches which strongly withstand the freeze-thaw cycles were further subjected for centrifugation test

\section{- Centrifugation test}

Centrifugation test was performed to access the physical instabilities of formulations. After the third cycle of freeze thaw, stable formulation systems weresubjected to centrifugation at $5000 \mathrm{rpm}$ for $20 \mathrm{mins}$ and observed for any signs of phase separation and drug precipitation. The formulation batches which show no sign of drug precipitation and phase separation were further evaluated for their robustness to dilution.

\section{- Robustness to dilution}

Robustness to dilution was studied by diluting liquid SMEDDS of UDL by 1000 times with dissolution media i.e 0.1N HCL, Phosphate buffer $\mathrm{pH} 6.8$ and water. The diluted microemulsions were stored for 12 hours at room temperature and observed for appearance of produced microemulsion, $\%$
$\mathrm{T}$ and for any signs of phase separation and/or drug precipitation.

Evaluation of optimized formulations of UDL L-SMEDDS

- Globule size, polydispersibility index and zeta potential

The L-SMEDDS, 50mg was diluted to $50 \mathrm{ml}$ with distilled water, SGF and SIF. The mean globule size, Polydispersity index (P.I.) and Zeta potential of the resulting microemulsion were determined by Horiba nanopartica SZ 100.

\section{- Effect of formulation matrix on UV} absorbance

It was observed that direct spectrophotometric method often suffer big disadvantages of their low selectivity and accuracy. Thus UV spectral scanning of drug containing and blank formulations were performed at wavelength range of 200-400 nm to detect any possible interference.

\section{- Drug content}

Accurately weighed L-SMEDDS equivalent to $10 \mathrm{mg}$ of UDE was placed in $10 \mathrm{~mL}$ volumetric flask and volume was made up with methanol, followed by sonication in bath sonicator for $15-20 \mathrm{~min}$ to extract and solublize the UDL. The concentration of UDL was determined by measuring $291.8 \mathrm{~nm}$ against methanol as blank, concentration of UDL was 
calculated using the calibration curve equation.

\section{- In vitro dissolution study}

In vitro dissolution of UDL API (50mg) and L-SMEDDS of UDL (equivalent to $50 \mathrm{mg}$ of UDL) filled in hard gelatin capsules were studied using I.P apparatus II at $37 \pm 0.5^{\circ} \mathrm{C}$ with a rotating speed of 75 rpm in media $0.1 \mathrm{~N}$ HCL [to Simulate Gastric Fluid (SGF)]. During the study, $5 \mathrm{~mL}$ of aliquots were removed at predetermined time intervals i.e. $0,5,10$, $15,20,25,30,45$ and $60 \mathrm{~min}$ from the dissolution medium and replaced with fresh buffer to maintain a sink condition. The amount of released drug was determined by measuring absorbance at $291.8 \mathrm{~nm}$. The dissolution study was performed in other dissolution media i.e phosphate buffer $\mathrm{pH}$ 6.8 [to simulate Intestinal fluid (SIF)], Acetate buffer $\mathrm{pH} 4$, distilled water to examine the effect of $\mathrm{pH}$ on drug release.

\section{Development of Super-SMEDDS formulation}

The maximum amount of drug that can be incorporated in optimized SMEDDS formulation was found to be $50 \mathrm{mg}$ due to its limited solubility in oil lipid phase. So, in order to produce the formulation comparable with the available marketed tablet (100mg), trials were taken for improving loading. To overcome this limitation the SMEDDS approach was further extended to the Super-SMEDDS. In Super-SMEDDS drug loading was increased by $100 \%$ i.e. $100 \mathrm{mg}$ of drug was solubilized in preconcentrates. For the preparation of supersaturated preconcentrates (Super-SMEDDS, drug above equilibrium solubility), drug load was increased by solubilizing more $50 \mathrm{mg}$ in SMEDDS preconcentrates which contain $50 \mathrm{mg}$ of drug, further they were subjected to a heating phase $40^{\circ} \mathrm{C}-50^{\circ} \mathrm{C}$ on heating mantle for 10-15 mins.

\section{Evaluation of super SMEDDS formulation}

Super-SMEDDS formulation was subjected to freeze thaw cycling, centrifugation and robustness to dilution study to evaluate any kind of instability, drug precipitation, phase separation.

- Globule size, polydispersibility index and zeta potentialSuper-SMEDDS, 50mg was diluted to $50 \mathrm{ml}$ with distilled water, SGF and SIF.The mean globule size, polydispersibility index (P.I.) and zeta potential of the resulting microemulsion were determined by Horiba nanopartica SZ 100.

\section{- Drug content}

Accurately weighed, Super-SMEDDS equivalent to $10 \mathrm{mg}$ of UDL was placed in $10 \mathrm{~mL}$ volumetric flask and volume was made up with methanol, followed by sonication in bath sonicator for $15-20 \mathrm{~min}$ 
to extract and solubilize the UDL. The concentration of UDL was determined by measuring absorbance at $291.8 \mathrm{~nm}$ against methanol as blank, concentration of UDL was calculated using the calibration curve equation.

\section{- In vitro dissolution study}

In vitro dissolution of plain UDL powder (100mg) and Super-SMEDDS of UDL (equivalent to $100 \mathrm{mg}$ of UDL) filled in hard gelatin capsules were studied using I.P apparatus II at $37 \pm 0.5^{\circ} \mathrm{C}$ with a rotating speed of $75 \mathrm{rpm}$ in media 0.1N HCL [to Simulate Gastric Fluid (SGF)]. During the study, $5 \mathrm{~mL}$ of aliquots were removed at predetermined time intervals i.e. 0, 5, 10, $1520,25,30,45$ and $60 \mathrm{~min}$ from the dissolution medium and replaced with fresh buffer to maintain a sink condition. The amount of released in the dissolution medium was determined by measuring absorbance at $291.8 \mathrm{~nm}$. The dissolution study was also performed in Phosphate buffer $\mathrm{pH} 6.8$ [to simulate Intestinal fluid (SIF)] to examine the effect of $\mathrm{pH}$ on drug release. Super-SMEDDS formulation were also compared with marketed tablet UDZIRE $(100 \mathrm{mg})$ in both SGF and SIF.

- Ex vivo intestinal permeability study by Everted sac technique of Krebs$\begin{array}{lll}\text { Ringer-phosphate-buffer } \quad \mathrm{pH} & \mathbf{7 . 2}\end{array}$ (KRPB perfusion solution)
Preparation of UDL solution and microemulsion of $U D L$

The UDL solution was prepared by dissolving $10 \mathrm{mg}$ of UDL in $100 \mathrm{~mL}$ of Krebs-Ringer's buffer solution to yield UDL concentration of $100 \mu \mathrm{g} / \mathrm{mL}$. UDL Super-SMEDDS equivalent to $10 \mathrm{mg}$ was added in to beaker containing $100 \mathrm{~mL}$ of Krebs-Ringer's buffer solution, the mixture was homogenized to produce fine microemulsion with the help of magnetic stirrer at $100 \mathrm{rpm}$ for $5 \mathrm{~min}$. The resultant microemulsion contains $100 \mu \mathrm{g} / \mathrm{mL}$ of UDL.

Transmittance study of resultant microemulsion in Krebs-Ringer's buffer solution: Robustness to dilution of SuperSMEDDS in Krebs-Ringer's buffer solution was assessed to determine the efficiency of Super-SMEDDS formulation to produce fine-microemulsion. This parameter was assessed by visual observation for appearance and measuring percent transmittance using Krebs-Ringer's buffer solution as blank.

Stability of UDL in Krebs-Ringer's buffer solution

Tightly closed volumetric flask containing plain UDL in Krebs-Ringer's buffer solution and microemulsion produced by diluting Super-SMEDDS in to KrebsRinger's buffer solution was stored at $37^{\circ} \mathrm{C}$ 
in incubator for the period of $2 \mathrm{~h}$. After $2 \mathrm{~h}$ of storage, solutions were visually

\section{Permeability study by Everted sac technique}

Exvivo study of Super-SMEDDS was carried out by using everted chicken intestinal sac. Chicken was killed and duodenal part of the small intestine was isolated and washed with distilled water to remove mucous and lumen content and them placed in cold KRPB solution continuously aerated with the help of electrical aerator. 5-6 cm long sac was prepared by tying the two ends of the sac ligated with silk thread to one end of a glass rod, and carefully everted on the glass rod. The everted gut sac, filled with $2 \mathrm{~mL}$ of KRPB solution was placed inside the $100 \mathrm{~mL}$ glass beaker containing $50 \mathrm{~mL}$ of either UDL solution or microemulsion of UDL Super-SMEDDS the KRPB solution, continuously bubbled with atmospheric air and maintaining the temperature $37 \pm 0.5$ ${ }^{\circ} \mathrm{C}$. The solution outside the sac was termed mucosal fluid, and the solution inside the sac was termed serosal fluid. An aliquot of drug solution was withdrawn from the serosal compartment after 15, 30, 60 mins. Volume of serosal fluid removed was noted. Amount of UDL from SuperSMEDDS microemulsion and Udenafil API solution permeated across the intestine (from mucosal surface to serosal sac) was determined using UV-Visible spectrophotometer after appropriate dilutions using suitable blank. Experiment was repeated in triplicate for each test solution containing UDL. Relative permeability was calculated using following formula, Relative permeability $\left(\mu \mathrm{g} / \mathrm{cm}^{2}\right)=$ Concentration of drug $\mu \mathrm{g} / \mathrm{mL}$ (in serosal fluid) X Volume $\mathrm{mL}$ (of serosac fluid) / Mucosal surface area, $\mathrm{cm}^{2}$

- Drug and Excipient compatibility study by DSC

Compatibility of UDL and selected excipients to produce L-SMEDDS was assessed by placing the mixture of UDL and each excipient in caped glass vials at room temperature for 30 days.

A thermal property of L-SMEDDS, Placebo L-SMEDDS, Super-SMEDDS was investigated using a SII Nanotechnology (SIEKO) DSC 6220. About 10mg of sample and alumina filled in aluminium pan in sample and control compartment of furnace. The heat runs was set from $50^{\circ} \mathrm{C}$ to $300^{\circ} \mathrm{C}$ with increment at $5^{\circ} \mathrm{C} / \mathrm{min}$, under an inert environment using nitrogen gas flow rate adjusted to $50 \mathrm{~mL} / \mathrm{min}$. The thermograph was recorded using Muse Measurement $6.9 \mathrm{U}$ software.

\section{RESULTS AND DISCUSSIONS}

Ultavioiet Spectroscopy Method for Estimation of $U D L$ 
It was observed that UDL in methanol shows maximum absorbance at wavelength of $291.8 \mathrm{~nm}$. Hence, $291.8 \mathrm{~nm}$ was selected as wavelength for estimation of udenafil. Values of Coefficient of correlation indicate a good correlation between the concentration range and absorbance studied. It indicates thatUDLfollows BeerLamberts law in the range of 5-35 $\mu \mathrm{g} / \mathrm{mlin}$ phosphate buffer 6.8 , Acetate buffer, $0.1 \mathrm{~N}$ $\mathrm{HCl}$ and water as shown in as shown in Figure 1.

Solubility Study of UDL in Water and Buffer Solutions

UDL solubility in water was found to be $0.06 \pm 0.005 \mathrm{mg} / \mathrm{ml}$. In accordance to report of the literatures, solubility of UDL in water is $0.0798 \mathrm{mg} / \mathrm{ml}$. However; water solubility does not represent the gastrointestinal tract conditions, mainly $\mathrm{pH}$ medium. Thus, to simulate these conditions, solubility was measured in SGF, SAF and SIF; suggested by USP for dissolution study of UDL. Solubility of UDL in SGF was observed to be $35.40 \pm$ $0.76 \mathrm{mg} / \mathrm{ml}$, in SAF was observed to be $21.10 \pm 0.51 \mathrm{mg} / \mathrm{ml}$ and in SIF was observed to be $1.71 \pm 0.45 \mathrm{mg} / \mathrm{ml}$ as shown in figure 2.

\section{Solubility Study of UDL in Various Oils}

Solubility studies were aimed at identifying suitable oily phase and surfactants for the development of UDL SMEDDS.
Identifying the suitable oil, surfactant/cosurfactant having maximal potential to solubilize the drug under investigation is very important to achieve optimum drug loading. Different oils were screened in preliminary trials to determine approximate solubility of UDL in each different oil phase by visual observation. Amongst the various different oil phases that were screened, it was found that only Oleic acid and Capmul MCM NF were able to solubilize an appreciable quantity of UDL as compared to other oils as shown in Table 1. Based on the approximate solubility, Oleic acid and Capmul MCM $\mathrm{NF}$ were further investigated and saturation solubility studies were performed by adding excess amount of UDENAFIL in selected oil.It was observed that oleic acid has high UDL solubilization capacity (355.32 $\pm 2.73 \mathrm{mg} / \mathrm{gm})$ followed by Capmul MCM NF $(34.56 \pm 1.51 \mathrm{mg} / \mathrm{gm})$. Therefore oleic acid was selected as oily phase produce L-SMEDDS of UDL due to its higher potential to solublize UDL and relative ease of microemulsification ability.

\section{Emulsification Efficiency Study}

Emulsification efficiency of varoius surfactants and cosurfactants was screened on the basis of \% Transparency andemulsification ability. The surfactants were screened for their emulsification efficiency for the selected oily phase i.e 
Oleic acid. It has been reported that well formulated SMEDDS is dispersed within seconds under gentle stirring conditions. Selected oily phase i.e oil and surfactant were explored for its emulsification ability in $1: 1$ ratio. It gave lower percent transmittance, so $1: 3$ ratio of surfactant: cosurfactant was investigated. On the basis of observations Cremophor RH-40 that requiredonly 3 flask inversions (3 FI) for homogenous transparent emulsion formation was ranked $1^{\text {st }}(97.10 \% \mathrm{~T})$ and Cremophor EL $(91.26 \% \mathrm{~T})$ was ranked $2^{\text {nd }}$ as itrequired 5 Flask inversions (5 FI). Other surfactants demonstrated poor emulsification efficiency with lower $\% \mathrm{~T}$. Results are shown in Table 2. Additionally, Cremophor RH 40 reported to have bioactive effects as they possess inhibitory effect on P-gp and CYP3A enzymes ${ }^{28}$. Based on these findings of emulsification efficiency study, it will be rational selection of Cremophor RH 40 as surfactant for further study to produce UDL SMEDDS that would positively contribute towards the oral bioavailability of UDL by decreasing its first pass metaboloism. CrRH40 is supposed to increase lipophilic drug bioavailability not only via solubilization theory but also due to bioactive respects [8].

\section{Selection of Co-surfactant}

Addition of a co-surfactant to the surfactant-containing formulation lowers the interfacial tension, fluidizes the hydrocarbon region of the interfacial film, and decreases the bending stress of interface which improves the dispersibility and drug absorption from the formulation. Various co-surfactants as given in Table 3 were investigated for spontaneity of emulsification for surfactant Cr-RH 40. PEG 400 showed superior performance and therefore PEG 400 is selected as cosurfactant to emulsify the oil phase i.e oleic acid which showed good spontaneity of emulsion formation.

\section{Construction of Ternary Phase Diagram}

Based on emulsification ability of surfactants and their spontaneity enhancement by co-surfactants systems were selected to plot ternary phase diagram to identify the microemulsion region. The phase diagrams of systems, Combination; Oleic acid: Cr-RH 40: PEG 400 are shown in Figure 3. The captions in the figures indicating the systems possessing ability to produce fine microemulsion, coarse Emulsion and turbid emulsion (no Emulsion). Observations were noted based on its visual appearance and \% transmittance values. It is likely reported that the systems with $100 \%$ transmittance indicates that the globule size lies in the nano range. From the phase diagram, it is 
evident that the system Oleic acid: $\mathrm{Cr}-\mathrm{RH}$ 40: PEG 400 possesses the ability to produce fine microemulsion $(>95 \% \mathrm{~T})$ till the oily phase (Oleic acid) concentration reaches to $25 \%(\mathrm{w} / \mathrm{w})$.

Effect of UDL loading and $p H$ of the aqueous phase on selected system OLA: Cr-RH 40: PEG 400

Ternary phase diagram mentioned in Figure 3 and illustrated the effect of UDL loading and $\mathrm{pH}$ of dilution medium on ternary phase diagram of combination Oleic acid: Cr-RH40: PEG 400. It was observed that loading of drug in oily phase and changing the $\mathrm{pH}$ of dilution medium does not show any significant change (like largely decrease in microemulsion area) on phase behaviour of this combination of excipients. As seen from ternary plot, in comparison to distilled water as a dilution medium, the microemulsion producing area was slightly decreased in SIF (pH 6.8) and slightly increase in SGF $(0.1 \mathrm{~N} \mathrm{HCl})$. These investigations confirm the ability of system OLA:Cr-RH40:PEG 400 to produce fine microemulsion of UDL on aqueous dilution irrespective of the $\mathrm{pH}$ of dilution media.

\section{Preparation of L-SMEDDS}

Based on the solubility profile, emulsification study and ternary phase of L-SMEDDS formulation systems of UDL were developed by changing the concentration ratio of Oil:Smix. Quantity of
UDL in all the formulation was kept constant $50 \mathrm{mg}$ per unit formula, at the same time the amount of oleic acid (150mg) was also kept constant which was selected based on the solubility profile of UDL in OLA. From forty five batches evaluated for their \% transmittance, four batches were selected that gave a good \% transmittance value. Compositions are given in Table 4.

\section{Optimization of UDL L-SMEDDS}

- Freeze Thaw cycling and centrifugation

SMEDDS are thermodynamically stable systems and are formed at a particular concentration of oil, surfactant and Cosurfactant, with no phase separation, creaming or cracking. The selected formulations were subjected to thermodynamic stress tests i.e freeze thaw cycle followed by centrifugation. Those formulations, which survived in thermodynamic stress tests, were evaluated further for their robustness to dilution study. All the four formulations systems (ULS1, ULS2, ULS3 and ULS4) were found to be stable at the end of freeze thaw cycles and there was no drug precipitation and phase separation even after centrifugation.

\section{- Robustness to dilution study}

Physical integrity of microemulsion formed and drug solublization capacity after 
dilution of SMEDDS must be assessed and ensured as it gives an idea about its performance in vivo. In view of this, UDL L-SMEDDS were diluted with aqueous phases differing in $\mathrm{pH}$. Effect of dilution and $\mathrm{pH}$ of dilution media on SMEDDS containing UDL is explained in Table 7. All the Four UDL L- SMEDDS spontaneously dispersed and formed microemulsion with less than 3 flask inversions and showed no sign of drug precipitation even after $12 \mathrm{~h}$ of dispersion. The clarity of microemulsions was assessed by transparency, measured in terms of transmittance $(\% \mathrm{~T})$. SMEDDS forms $\mathrm{o} / \mathrm{w}$ microemulsion since water is external phase. Out of four batches, formulation ULS3 was found robust to all dilutions as $\%$ transmittance value greater than 95\% and appeared clear or slightly bluish, secondly there was no drug precipitation observed even after $12 \mathrm{~h}$ of dispersion irrespective of $\mathrm{pH}$ of dilution media. Results are given in Table 5.

- Globule size, Polydispersibility index (P.I), Zeta potential analysis

The globule size of the emulsion is a crucial factor for self-emulsification performance because it determines the rate and extent of drug release as well as drug absorption. Also, it has been reported that the smaller globule size of the emulsion droplets may lead to more rapid absorption and improve the bioavailability [9]. The mean globule size obtained from optimized L-SMEDDS of UDL was in the range of 145-160 nm. The globule size was observed as $158.8 \pm 2.15 \mathrm{~nm}$ in SGF, 145.2 $\pm 1.28 \mathrm{~nm}$ in distilled water (Figure 4) and $151.1 \pm 3.23 \mathrm{~nm}$ in SIF. Polydispersibility is the ratio of standard deviation to the mean droplet size. This signifies the uniformity of droplet size within the formulation. The higher the value of polydispersibility, the lower is the uniformity of the droplet size in the formulation. The polydispersibility index of UDL L-SMEDDS in all three media was in the range of 0.369 to 0.428 . A dividing line between stable and unstable aqueous dispersions is generally taken at either $\pm 30 \mathrm{mV}$. Particles with zeta potentials more negative than $-30 \mathrm{mV}$ are normally considered stable [10]. Zeta potential value obtained was $-39 \mathrm{mV}$ (Figure 5). These findings indicate that, the optimized UDL L-SMEDDS (ULS3) produced fine microemulsion with a small mean size and a narrow particle size distribution. Results are given in Table 6.

- Effect of formulation matrix on UV absorbance

No interference was observed between UDL and excipients selected at the wavelength of estimation of UDL (Figure 5).

- Drug Content 
Drug content of optimized L-SMEDDS was determined using UV spectrophotometer and it was observed to be $98.9 \pm 0.49 \%$. Results suggested that UDL is completely dissolved and uniformly distributed in self-emulsifying system.

\section{- In-Vitro Dissolution study}

In-vitro dissolution profiles, of optimized UDL L-SMEDDS (ULS3) and UDL API filled in hard gelatin capsule, in water, $\mathrm{pH}$ 4(SAF), pH 6.8(SIF) and 0.1N HCl (SGF) are shown in Figure 6. It is evident from the observation that UDL L-SMEDDS showed a dramatic improvement in the in vitro dissolution profile compared to the UDL API in all four dissolution media viz. Water, 0.1N HCl (SGF), pH 6.8 (SIF), pH 4(SAF). L-SMEDDS formulations were able to release and solubilise udenafil completely in different $\mathrm{pH}$ media but UDL API showed release to a greater extent in $0.1 \mathrm{~N} \mathrm{HCL} \mathrm{(SGF).} \mathrm{It} \mathrm{is} \mathrm{concluded} \mathrm{that}$ release from API was found to be $\mathrm{pH}$ dependent as udenafil is a pH-dependent drug, while the dissolution from LSMEDDS of udenafil was not affected by varying $\mathrm{pH}$ range of the GIT [11]. This result suggests that there is better absorption of udenafil in the GIT. The barrier in absorption of udenafil i.e poor dissolution in gastrointestinal environment with higher $\mathrm{pH}$ was overcome as the dissolution of SMEDDS loaded drug was completely independent of media $\mathrm{pH}$. UDL is classified as Class-II drugs as per BCS classification system, and it is well known that its poor water solubility limits its dissolution and hence leads to low oral bioavailability. Optimized L-SMEDDS presents the UDL in dissolved form and produce fine globule which disperse spontaneously irrespective of the $\mathrm{pH}$ of dissolution media. The rate and extent of UDL release from L-SMEDDS suggested that it may improve the oral bioavailability of UDL.

\section{Development of super SMEDDS formulation}

Super-SMEDDS were prepared by increasing drug load. The composition of the optimised formulation is given in Table 7.

Freeze thaw cycling, Centrifugation test, Robustness to dilution

Super-SMEDDS formulation wasevaluated for instability, drug precipitation and phase separation by subjecting it to freeze thaw cycling, centrifugation and robustness to dilution test. No phase separation, drug precipitation was found in super-SMEDDS formulation. Results are given in Table 8.

Globule size, Polydispersibility index (P.I), Zeta potential analysis, Drug content

The mean globule size obtained from optimized Super-SMEDDS of UDL was in 
the range of 189-202 $\mathrm{nm}$. The globule size was observed as $201.9 \pm 1.8 \mathrm{~nm}$ in SGF, $189.1 \pm 1.1$ in DW and $198.16 \pm 2.3$ in SIF. The polydispersibility index of UDL LSMEDDS in various media was in the range of 0.308 to 0.384 . Zeta potential value obtained was $-39.1 \mathrm{mV}$. Drug content was found to be $98.9 \pm 0.58 \%$. Results are given in Table 9.

\section{In-vitro dissolution study}

In-vitro dissolution profiles, of optimized UDL Super-SMEDDS (ULS $\left.{ }_{3}\right)$ marketed Udenafil tablet (Udzire 100mg), in SGF and SIF are shown in fig 7 . It is evident from the observation that UDL SuperSMEDDS showed a dramatic improvement in the in-vitro dissolution profile compared to the UDL API and Udzire tablet in $\mathrm{pH}$ 6.8(SIF).

Ex vivo drug permeability study using everted sac techniques

\section{- Transmittance study}

Percent transmittance value of resultant emulsion was observed to be 96.56.It indicates that Super-SMEDDS produced fine-microemulsion in Krebs-Ringer buffer solution.

-Stability of UDL in Krebs-Ringer buffer solution

There was no precipitation of UDL in UDL solution and microemulsion before and after the storage for two hours in incubator. No significant changes, in UDL concentration in the Krebs-Ringer buffer solution was observed before and after two hours of storage at $37^{\circ} \mathrm{C}$. This result indicates that UDL was stable in KrebsRinger buffer solution.

\section{-Permeability of UDL}

Significant increase in permeability of UDL was observed from Super-SMEDDS as compared to UDL API. After $60 \mathrm{~min}$ of study it was observed that, only $25 \%$ of UDL was transported through intestinal lumen from UDL solution, while $75 \%$ of UDL was transported through intestinal lumen from microemulsion produced from Super-SMEDDS, with nearly three fold improvement in permeability. Results are shown table 10. This 3 fold improvement of permeability of UDL from Super-SMEDDS was attributed by many reasons, mainly the uniformly dispersed globules having nano size in which UDL is present in the dissolved state. These globule size increases the surface area which facilitates the permeability of drug.

Drug and Excipient compatibility study by visual inspection and DSC

Visual observations of each mixture suggested that there was no change in color and appearance even after 30 days of study. DSC thermogram of UDL showed one peak at $161.7^{\circ} \mathrm{C}$ representing melting peak of API. DSC thermogram of L-SMEDDS, Placebo L-SMEDDS show one peak at 
$100^{\circ} \mathrm{C}$. DSC thermogram of Super- that drug is in a molecularly dissolved state SMEDDS shows one peak at $98^{\circ} \mathrm{C}$. Super-SMEDS [12]. Thermograms are

Absence of melting peak in thermogram of shown in Figure 8.

UDL in developed formulation indicates

Table 1: Approximate estimated solubility of UDL in various oily phases

\begin{tabular}{|c|c|c|c|c|c|c|c|c|}
\hline \multirow{2}{*}{$\begin{array}{c}\text { Sr. } \\
\text { No. }\end{array}$} & \multirow[t]{2}{*}{ Oil } & \multicolumn{5}{|c|}{ Quantity of drug added } & \multirow[t]{2}{*}{ Visual observation } & \multirow{2}{*}{$\begin{array}{c}\text { Approx. } \\
\text { Solubility } \\
\text { (mg/gm) }\end{array}$} \\
\hline & & $10 \mathrm{mg}$ & $10 \mathrm{mg}$ & $10 \mathrm{mg}$ & $10 \mathrm{mg}$ & $10 \mathrm{mg}$ & & \\
\hline 1. & Corn oil & $\checkmark$ & & & & & Slightly Soluble & $\leq 10$ \\
\hline 2. & Sunflower oil & $\checkmark$ & & & & & Slightly Soluble & 10 \\
\hline 3. & Olive oil & $\checkmark$ & & & & & Slightly Soluble & 10 \\
\hline 4. & Castor oil & $\checkmark$ & & & & & Slightly Soluble & $\leq$ \\
\hline 5. & Coconut oil & $\checkmark$ & $\checkmark$ & & & & Soluble & $\geq$ \\
\hline 6. & Soyabean oil & $\checkmark$ & & & & & Slightly Soluble & $\leq$ \\
\hline 7. & Arachis oil & $\checkmark$ & & & & & Slightly Soluble & $\leq \mathbf{1 0}$ \\
\hline 8. & Tea tree oil & $\checkmark$ & $\checkmark$ & $\checkmark$ & $\checkmark$ & $\checkmark$ & Highly Soluble & $\geq 160$ \\
\hline 9. & Isopropyl myristate & $\checkmark$ & & & & & Slightly Soluble & $\leq 10$ \\
\hline 10. & Captex 355 & $\checkmark$ & & & & & Slightly Soluble & $\leq$ \\
\hline 11. & Captex 200 & $\checkmark$ & & & & & Slightly Soluble & $\leq$ \\
\hline 12. & Captex $355 \mathrm{EP} / \mathrm{NF}$ & $\checkmark$ & & & & & Slightly Soluble & $\leq$ \\
\hline 13. & Labrafac PG & $\checkmark$ & & & & & Slightly Soluble & $\leq$ \\
\hline 14. & Capmul MCM NF & $\checkmark$ & $\checkmark$ & $\checkmark$ & & & Soluble & $\geq$ \\
\hline 15. & Capmul MCM C8 & $\checkmark$ & $\checkmark$ & & & & Soluble & $\geq$ \\
\hline 16. & Capmul MCM EP & $\checkmark$ & $\checkmark$ & & & & Soluble & $\geq 20$ \\
\hline 17. & Oleic acid & $\checkmark$ & $\checkmark$ & $\checkmark$ & $\checkmark$ & $\checkmark$ & Highly Soluble & $\geq 350$ \\
\hline
\end{tabular}

Table 2: Emulsification ability of surfactants using oil to surfactant ratio of 1:1 \& 1:2

\begin{tabular}{|c|c|c|c|c|}
\hline Sr. No. & Surfactant & No. of Flask Inversion & $\% \mathrm{~T}$ & Appearance \\
\hline \multicolumn{5}{|c|}{ 1:1 ratio of oil:surfactant } \\
\hline 1. & Cremophor RH 40 & 10 & 67.45 & Turbid \\
\hline 2. & Cremophor EL & 10 & 58.11 & Turbid \\
\hline 3. & Tween 80 & 10 & 42.07 & Turbid \\
\hline 4. & Tween 20 & 10 & 43.15 & Turbid \\
\hline 5. & Span 20 & 13 & 26.83 & Turbid \\
\hline 6. & Span 80 & 10 & 30.15 & Turbid \\
\hline 7. & Polaxomer P188 & 10 & 74.27 & Colloidal \\
\hline 8. & Polaxomer P407 & 10 & 78.97 & Colloidal \\
\hline 9. & Labrafil M2130C5 & 15 & 27.88 & Turbid \\
\hline 10. & Labrafil LWL1349 & 15 & 46.67 & Turbid \\
\hline 11. & Cremophor TPGS & 15 & 13.20 & Turbid \\
\hline 12. & Solutol HS 15 & 20 & 9.05 & Turbid \\
\hline 13. & Labrafil M 1944 CS & 12 & 38.73 & Turbid \\
\hline 14. & Labrafil M2125CS & 15 & 26.12 & Turbid \\
\hline 15. & Labrasol ALF & 15 & 28.45 & Turbid \\
\hline \multicolumn{5}{|c|}{ 1:3 ratio of oil:surfactant } \\
\hline 16. & Cremophor RH 40 & $\mathbf{3}$ & 97.10 & Clear \\
\hline 17. & Cremophor EL & 5 & 91.26 & Clear \\
\hline 18. & Tween 20 & 6 & 70.68 & Turbid \\
\hline 19. & Tween 80 & 7 & 73.58 & Turbid \\
\hline 20. & Span 20 & 13 & 46.83 & Turbid \\
\hline 21. & Span 80 & 10 & 60.15 & Turbid \\
\hline 22. & Polaxomer P188 & 10 & 77.27 & Colloidal \\
\hline 23. & Polaxomer P407 & 10 & 79.97 & Colloidal \\
\hline 24. & Labrafil M2130C5 & 15 & 47.88 & Turbid \\
\hline 25. & Labrafil LWL1349 & 15 & 66.67 & Turbid \\
\hline 26. & Cremophor TPGS & 15 & 23.20 & Turbid \\
\hline 27. & Solutol HS 15 & 20 & 20.05 & Turbid \\
\hline 28. & Labrafil M 1944 CS & 12 & 48.73 & Turbid \\
\hline 29. & Labrafil M2125CS & 15 & 46.12 & Turbid \\
\hline 30. & Labrasol ALF & 15 & 38.45 & Turbid \\
\hline
\end{tabular}


Table 3: Spontaneity of emulsification by co-surfactants for surfactant $\mathrm{Cr}-\mathrm{RH} 40$

\begin{tabular}{|c|c|c|c|}
\hline \multirow{2}{*}{ Co-surfactant } & \multicolumn{3}{|c|}{ Cremophor RH 40 } \\
\cline { 2 - 4 } & No. of flask inversion & \% T & Appearance \\
\hline Ethanol & 3 & 92.34 & Clear \\
\hline Propylene glycol & 3 & 92.51 & Clear \\
\hline Transcutol HP & 3 & 91.67 & Clear \\
\hline Labrafil 1944 CS & 8 & 90.99 & Clear and bluish \\
\hline PEG 400 & 3 & 95.45 & Colloidal \\
\hline Labrafil M 2125 CS & 10 & 77.54 & \\
\hline
\end{tabular}

Table 4: Formulation batches of UDL L-SMEDDS (ULS)

\begin{tabular}{|c|c|c|c|c|}
\hline \multirow{2}{*}{$\begin{array}{c}\text { Ingredients } \\
\text { (mg) }\end{array}$} & \multicolumn{4}{|c|}{ Batch No. } \\
\cline { 2 - 5 } & ULS $_{\mathbf{1}}$ & ULS $_{\mathbf{2}}$ & ULS $_{\mathbf{3}}$ & ULS $_{\mathbf{4}}$ \\
\hline UDL & $\mathbf{5 0}$ & $\mathbf{5 0}$ & $\mathbf{5 0}$ & $\mathbf{5 0}$ \\
\hline Oleic acid & 150 & 150 & 150 & $\mathbf{1 5 0}$ \\
\hline Cr RH 40 & $\mathbf{6 0 0}$ & $\mathbf{5 5 0}$ & $\mathbf{5 0 0}$ & $\mathbf{4 5 0}$ \\
\hline PEG 400 & $\mathbf{2 5 0}$ & $\mathbf{3 0 0}$ & $\mathbf{3 5 0}$ & $\mathbf{4 0 0}$ \\
\hline Total & $\mathbf{1 0 5 0}$ & $\mathbf{1 0 5 0}$ & $\mathbf{1 0 5 0}$ & $\mathbf{1 0 5 0}$ \\
\hline
\end{tabular}

Table 5: Data of robustness to dilution study for UDL L-SMEDDS

\begin{tabular}{|c|c|c|c|c|c|}
\hline \multirow{2}{*}{$\begin{array}{l}\text { Batch } \\
\text { No. }\end{array}$} & \multirow[t]{2}{*}{ Dilution Media } & \multirow[t]{2}{*}{ Dilution } & \multicolumn{3}{|c|}{ Evaluation parameters } \\
\hline & & & $\% \mathrm{~T}$ & Appearance & $\begin{array}{c}\text { Drug } \\
\text { precipitation }\end{array}$ \\
\hline \multirow[t]{3}{*}{$\mathbf{U L S}_{1}$} & D.W & \multirow[b]{3}{*}{1000} & 94.78 & Clear & No \\
\hline & 0.1N HCL (SGF) & & 93.21 & Clear & No \\
\hline & Phosphate buffer (SIF) & & 92.10 & Clear & No \\
\hline \multirow[t]{3}{*}{$\mathbf{U L S}_{2}$} & D.W & \multirow[b]{3}{*}{1000} & 96.3 & Clear & No \\
\hline & 0.1N HCL (SGF) & & 89.6 & Clear & No \\
\hline & Phosphate buffer (SIF) & & 94 & Clear & No \\
\hline \multirow[t]{3}{*}{$\mathbf{U L S}_{3}$} & D.W & \multirow[b]{3}{*}{1000} & 96.6 & Clear & No \\
\hline & 0.1N HCL (SGF) & & 95.8 & Clear & No \\
\hline & Phosphate buffer (SIF) & & 95.5 & Clear & No \\
\hline \multirow[t]{3}{*}{$\mathbf{U L S}_{4}$} & D.W & \multirow[b]{3}{*}{1000} & 89.3 & Clear & No \\
\hline & 0.1N HCL (SGF) & & 89.11 & Clear & No \\
\hline & Phosphate buffer (SIF) & & 89.21 & Clear & No \\
\hline
\end{tabular}

Table 6: Data representing Globule size, zeta potential, polydispersibility index of UDL L-SMEDDS in various dilution media

\begin{tabular}{|c|c|c|c|c|c|c|c|c|}
\hline \multicolumn{3}{|c|}{ Distilled water } & \multicolumn{3}{|c|}{$\begin{array}{c}\text { 0.1N HCl } \\
\text { (SGF) }\end{array}$} & \multicolumn{3}{|c|}{ Phosphate buffer pH 6.8 (SIF) } \\
\hline $\begin{array}{c}\text { Globule } \\
\text { Size (nm) }\end{array}$ & P.I. & $\begin{array}{c}\text { Zeta } \\
\text { potential } \\
(\mathrm{mV})\end{array}$ & $\begin{array}{c}\text { Globule } \\
\text { Size (nm) }\end{array}$ & P.I. & $\begin{array}{c}\text { Zeta } \\
\text { potential } \\
(\mathbf{m V})\end{array}$ & $\begin{array}{c}\text { Globule } \\
\text { Size (nm) }\end{array}$ & P.I. & $\begin{array}{c}\text { Zeta } \\
\text { potential } \\
(\mathrm{mV})\end{array}$ \\
\hline $\begin{array}{c}145.2 \pm \\
1.28\end{array}$ & 0.385 & -37.0 & $\begin{array}{c}158.8 \pm \\
2.15\end{array}$ & 0.369 & -31.5 & $\begin{array}{c}151.1 \pm \\
3.23\end{array}$ & 0.428 & -36.5 \\
\hline
\end{tabular}

Table 7: Formulation batch of UDL Super-SMEDDS

\begin{tabular}{|c|c|}
\hline Ingredients (mg) & Super-SMEDDS ULS \\
\hline UDL & 100 \\
\hline Oleic acid & 150 \\
\hline Cr-RH 40 & 500 \\
\hline PEG 400 & 350 \\
\hline Total & 1100 \\
\hline
\end{tabular}

Table 8: Evaluation of UDL Super-SMEDDS by freeze thaw cycling, Centrifugation, Robustness to dilution test

\begin{tabular}{|c|c|c|c|}
\hline \multirow{2}{*}{ Tests Performed } & \multicolumn{2}{|c|}{ Evaluation parameters } & \multirow{2}{*}{ Remark } \\
\cline { 2 - 3 } & Phase separation & Drug precipitation & \\
\hline 1. Freeze thaw cycling & Stable & No & Passes \\
\hline 2. Centrifugation & Stable & No & Passes \\
\hline 3. Robustness to dilution & Stable & No & Passes \\
\hline
\end{tabular}


Table 9: Data representing globule size, zeta potential, polydispersibility index of UDL Super-SMEDDS in various

\begin{tabular}{|c|c|c|c|c|c|c|c|c|}
\hline \multicolumn{9}{|c|}{ dilution media } \\
\hline \multicolumn{3}{|c|}{ Distilled water } & \multicolumn{3}{|c|}{$\begin{array}{c}\text { 0.1N HCl } \\
\text { (SGF) }\end{array}$} & \multicolumn{3}{|c|}{ Phosphate buffer pH 6.8 (SIF) } \\
\hline $\begin{array}{l}\text { Globule } \\
\text { Size (nm) }\end{array}$ & P.I. & $\begin{array}{c}\text { Zeta } \\
\text { potential } \\
(\mathrm{mV})\end{array}$ & $\begin{array}{l}\text { Globule } \\
\text { Size (nm) }\end{array}$ & P.I. & $\begin{array}{c}\text { Zeta } \\
\text { potential } \\
(\mathrm{mV})\end{array}$ & $\begin{array}{l}\text { Globule } \\
\text { Size }(\mathbf{n m})\end{array}$ & P.I. & $\begin{array}{c}\text { Zeta } \\
\text { potential } \\
(\mathrm{mV}) \\
\end{array}$ \\
\hline $189.1 \pm 1.1$ & 0.384 & -39.1 & $201.9 \pm 1.8$ & 0.308 & -33.3 & $\begin{array}{c}198.16 \pm \\
2.3\end{array}$ & 0.317 & -34.8 \\
\hline
\end{tabular}

Table 10: Data representing ex-vivo permeability of Super-SMEDDS UDL microemulsion and API UDL solution.

\begin{tabular}{|c|c|c|c|}
\hline \multirow{2}{*}{ Sr.no } & $\begin{array}{c}\text { Permeability assessment } \\
\text { period }\end{array}$ & API UDL solution & $\begin{array}{c}\text { Super-SMEDDS UDL } \\
\text { microemulsion }\end{array}$ \\
\cline { 3 - 4 } & & & $\mathbf{3 7 . 9} \pm 1.12$ \\
\hline 1. & $15 \mathrm{~min}$ & $\mathbf{1 0 . 1} \pm \mathbf{0 . 2 3}$ & $\mathbf{5 2 . 5} \pm \mathbf{0 . 5 9}$ \\
\hline 2. & $\mathbf{3 0} \mathrm{min}$ & $\mathbf{1 6 . 8} \pm 0.34$ & $\mathbf{7 5 . 9} \pm \mathbf{0 . 5 6}$ \\
\hline 3. & $60 \mathrm{~min}$ & $\mathbf{2 5 . 4} \pm 1.1$ & \multicolumn{2}{c|}{} \\
\hline
\end{tabular}

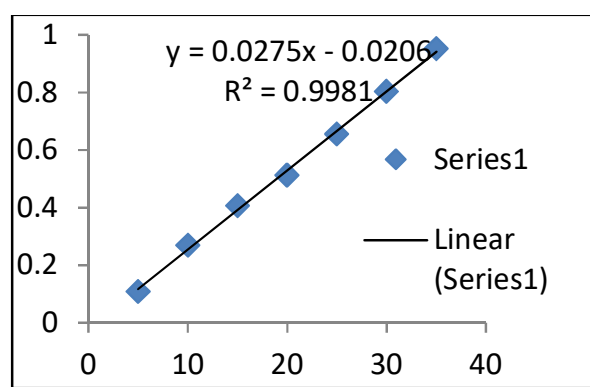

(a)

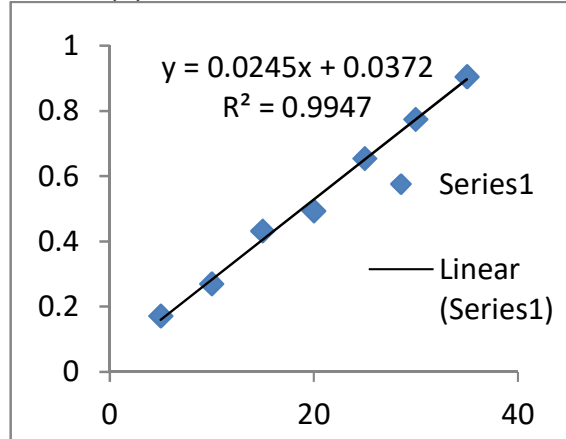

(c)

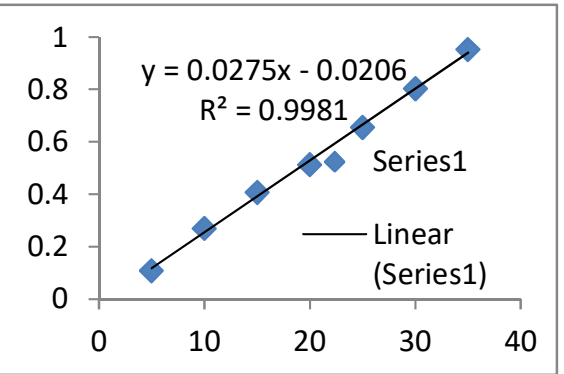

(b)

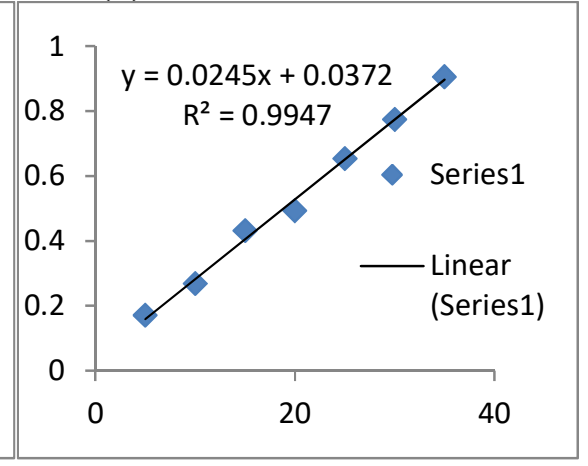

(d)

Figure 1: Calibration curve of UDL in phosphate buffer 6.8(a), Acetate buffer (b), $0.1 \mathrm{~N} \mathrm{HCl}(\mathrm{c})$ and water(d) 


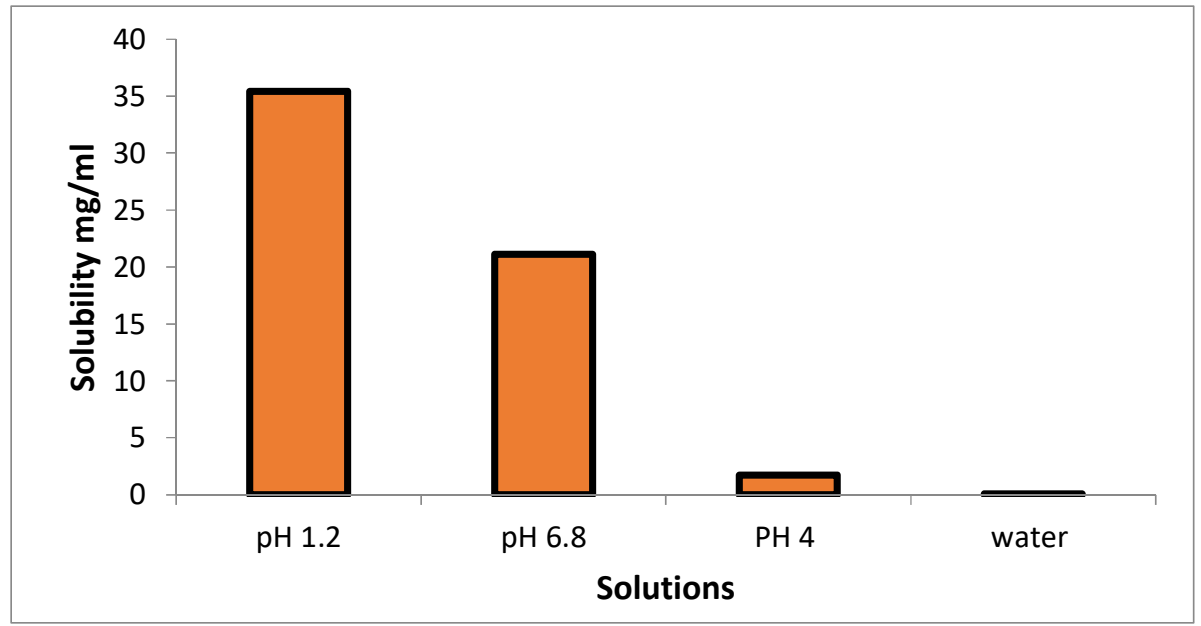

Figure 2: Solubility profile of UDL in Buffer solutions

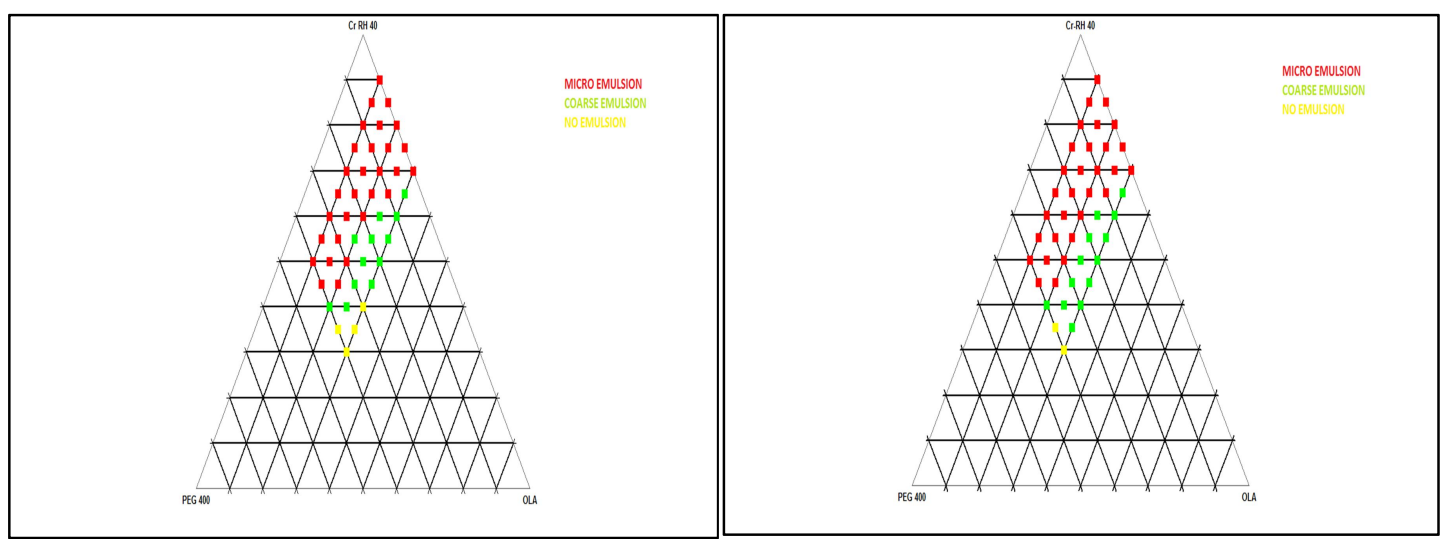

(a)

(b)

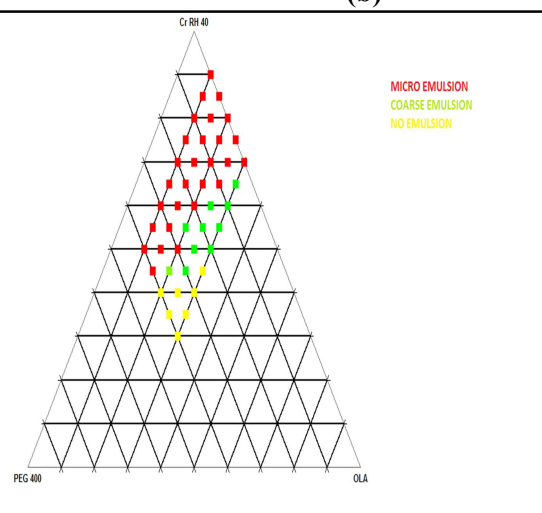

(c)

Figure 3: Ternary phase diagram for drug loaded system (Oleic acid: Cr-RH 40: PEG 400) in distilled water (a), SGF (b) and $\operatorname{SIF}(c)$ 


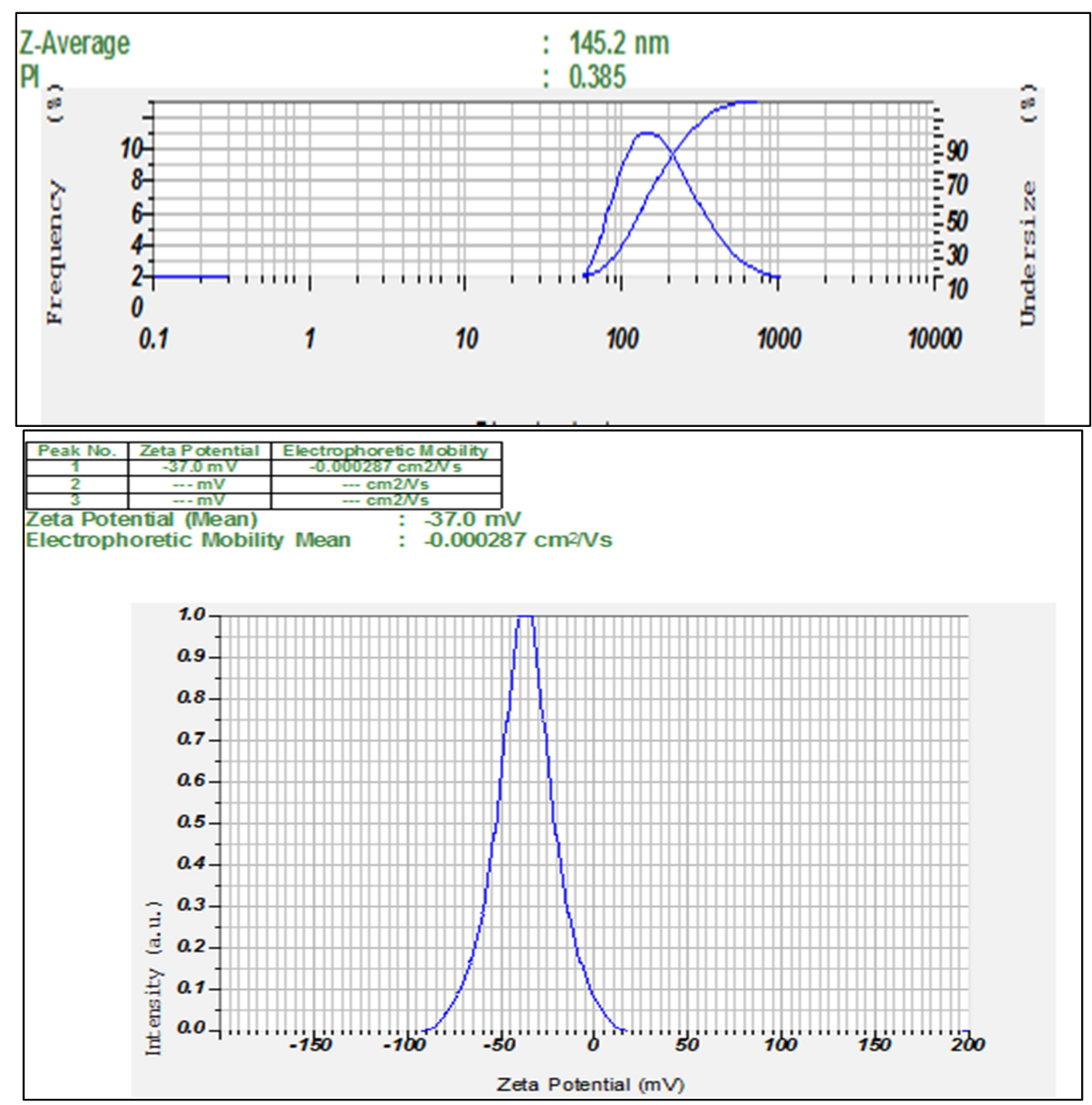

Figure 4: Globule size, P.I and zeta potential obtained from L-SMEDDS

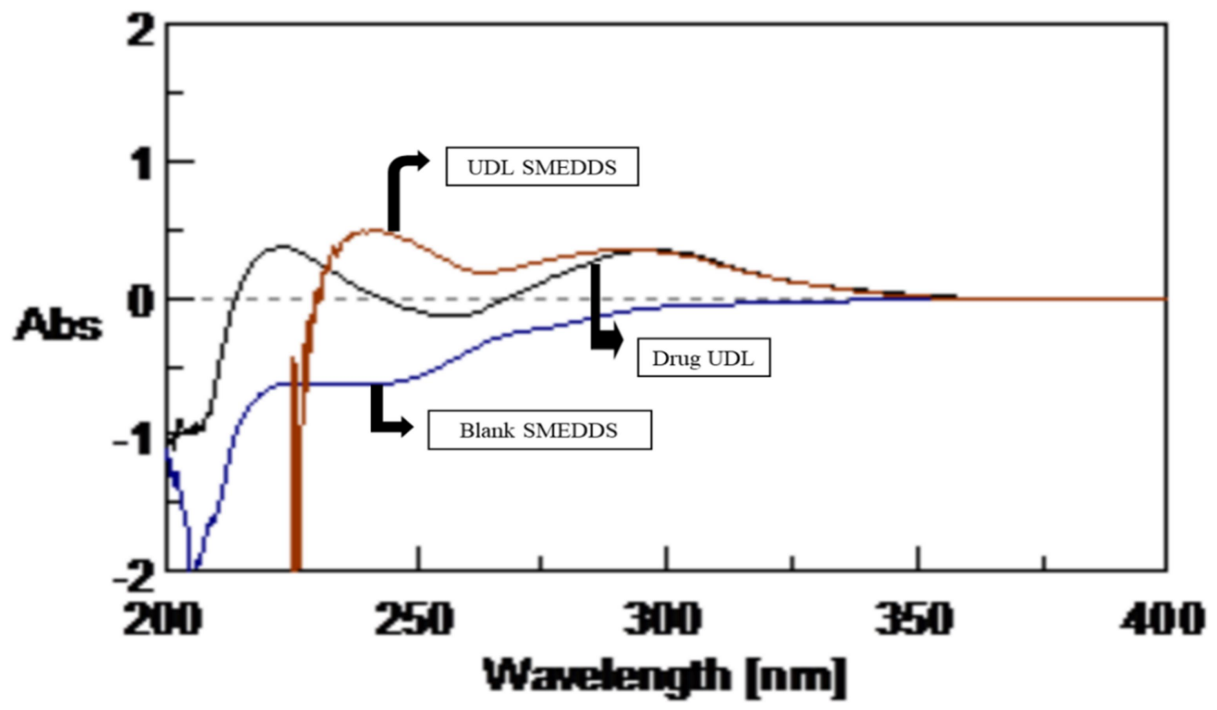

Figure 5: Overlay spectra of placebo SMEDDS, pure drug UDL, UDL loaded SMEDDS at $291.8 \mathrm{~nm}$ 


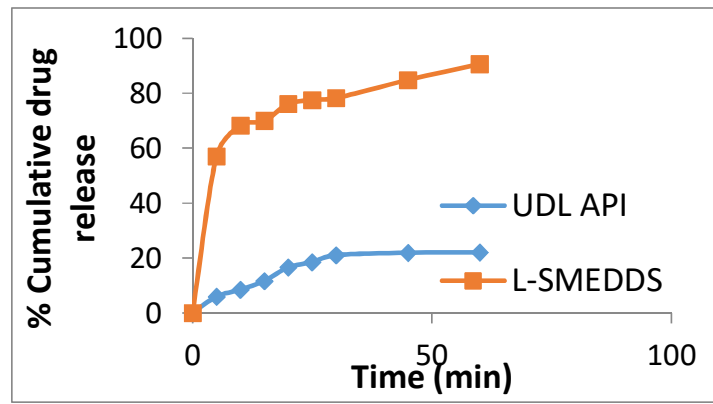

(a)

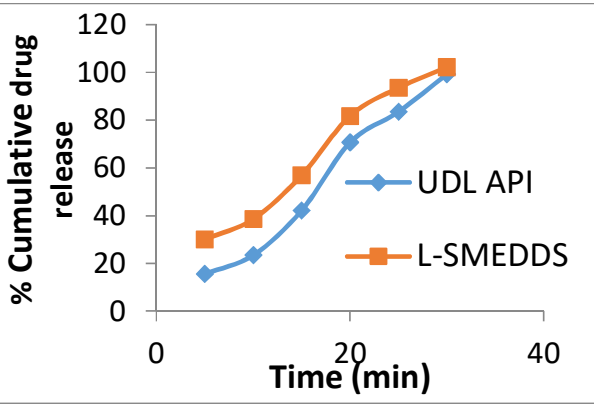

(b)

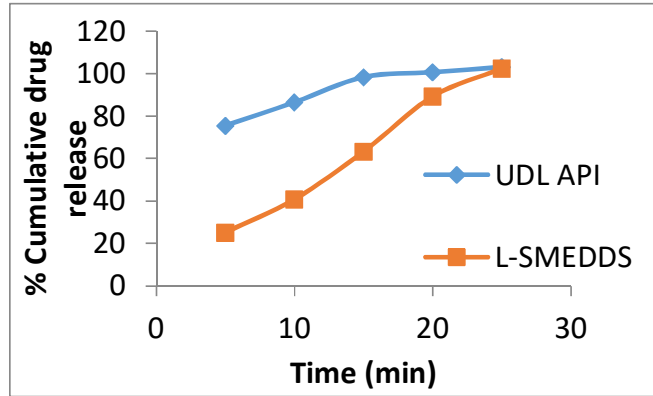

(c)

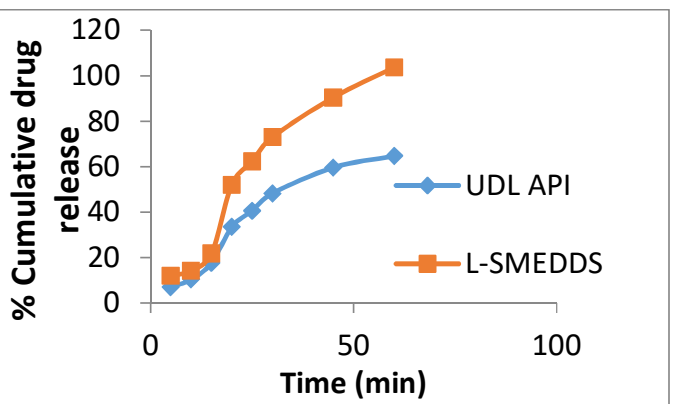

(d)

Figure 6: In-vitro drug release studies of developed SMEDDS formulation and UDL API in distilled water (a), acetate buffer(b), phosphate buffer pH (1.2) (c) and phosphate buffer pH 6.8 (d)

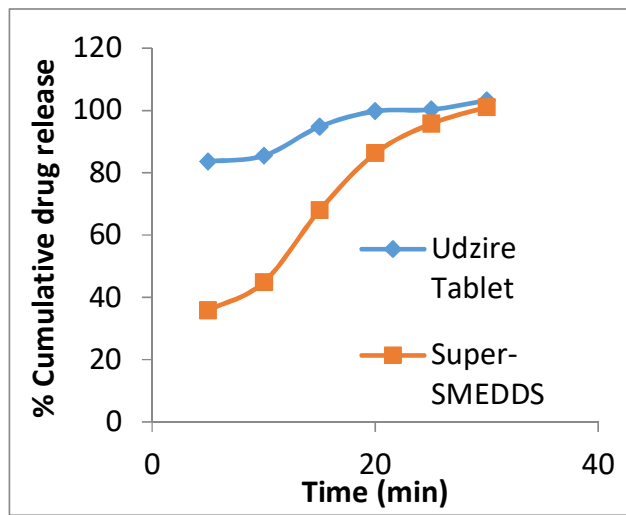

(a)

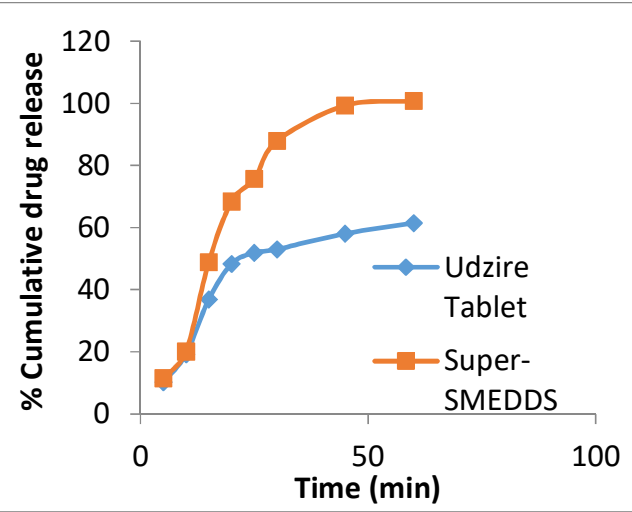

(b)

Figure 7: In-vitro drug release studies of developed super SMEDDS formulation and Udzire tablets in phosphate buffer pH (1.2) (a) and phosphate buffer pH 6.8 (b) 


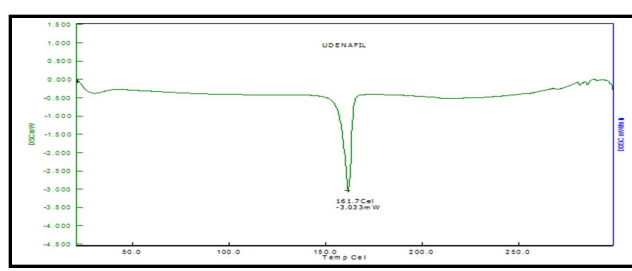

(a)

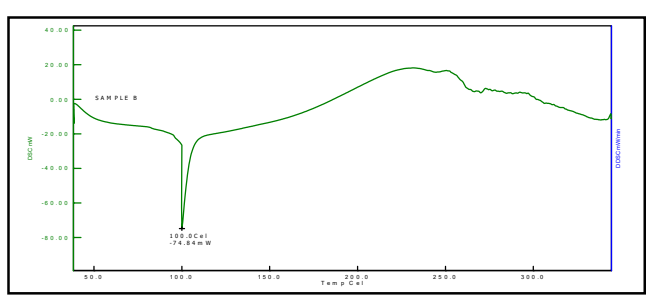

(b)

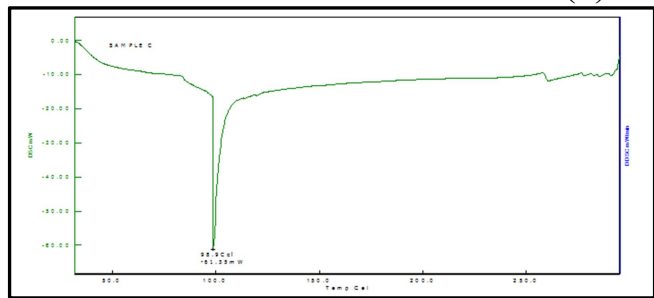

(c)

Figure 8: DSC Thermograms of drug(a), placebo L-SMEDDS(b) and drug loaded super- SMEDDS(c)

\section{Acknowledgement}

Authors are thankful to the principal and management of Dr. L. H. Hiranandani College of Pharmacy, Mumbai University for providing the necessary facilities. Authors are also thankful to Watson Pharma Pvt. Ltd for providing gift sample of Udenafil and Gattefosse, Mumbai, BASF, Mumbai for providing gift samples of oils and surfactants.

\section{REFERENCES}

[1] KalepuS, Manthina M, \& Padavala V (2013). Oral lipid-based drug delivery systems-an overview. Acta

Pharmaceutica Sinica B, 3(6): 361-372.

[2] SheikhZ \& Morshed N (2014). Optimizing oral drug delivery using lipid based formulations. International Research Journal of Pharmacy.

[3] Singh M A (2015). Self emulsifying systems: A review. Asian Journal of Pharmaceutics (AJP): Free full text articles from Asian J Pharm, 9(1): 1318.

[4] Sarpal K, PawarY B, \& Bansal AK. (2010). Self-emulsifying drug delivery systems: a strategy to improve oral bioavailability. Crips, 11(3): 42-49.

[5] ThomasN, HolmR, Müllertz, A., \& Rades, T. (2012). In vitro and in vivo performance of novel supersaturated self-nanoemulsifying drug delivery systems (super-SNEDDS). Journal of controlled release, 160(1): 25-32.

[6] Thomas N, Holm R, GarmerM, Karlsson J J, MüllertzA \& RadesT. (2013). Supersaturated selfnanoemulsifying drug delivery systems (Super-SNEDDS) enhance the bioavailability of the poorly watersoluble drug simvastatin in dogs. The AAPS journal, 15(1): 219-227.

[7] Rahul T, Vibhuti D \& Karthikeyan K (2019) Ocular Self-Microemulsifying 
Drug Delivery System of Prednisolone Improves Therapeutic Effectiveness in the Treatment of Experimental Uveitis, Ocular Immunology and Inflammation, 27(2): 303-311

[8] Wadhwa J, NairA, \& Kumria R. (2012). Emulsion forming drug delivery system for lipophilic drugs. Acta Pol Pharm, 69(2): 179-91.

[9] Hyma P. (2014). Formulation and characterization of novel self micro emulsifying drug delivery system of glimepiride. International journal of Science and Technology, 24(1): 16401648.

[10] Jaiswal P, AggarwalG, Harikumar S L, \& SinghK. (2014). Development of self-microemulsifying drug delivery system and solid-selfmicroemulsifying drug delivery system of telmisartan. International journal of pharmaceutical investigation, 4(4): 195.

[11]Lawrence M J, \& Rees G D(2000). Microemulsion-based media as novel drug delivery systems. Advanced drug delivery reviews, 45(1):89-121.

[12]Kim D S, Cho J H, Park J H, Kim J S, SongE S, KwonJ, Giri B R, JinS G, Kim K S, Choi H G, \& Kim D $\mathrm{W}(2019)$. Self-microemulsifying drug delivery system (SMEDDS) for improved oral delivery and photostability of methotrexate. International journal of nanomedicine, 14: 4949-4960. 